\title{
Accumulation of fructose 1,6-bisphosphate protects clear cell renal cell carcinoma from oxidative stress
}

\author{
Jun Wang ${ }^{1} \cdot \mathrm{Qi} \mathbf{W u}^{1,2} \cdot$ Jianxin $^{\mathrm{Qi}}{ }^{1}$
}

Received: 24 October 2018 / Revised: 19 December 2018 / Accepted: 4 January 2019 / Published online: 13 February 2019

(c) United States \& Canadian Academy of Pathology 2019

\begin{abstract}
Clear cell renal cell carcinoma (ccRCC) is characterized by the activation of hypoxia-inducible factors and enhanced aerobic glycolysis. In our previous study, metabolic profiling revealed a threefold increase of fructose 1,6-bisphosphate (FBP) in ccRCC tissue compared with normal kidney tissue. As an important intermediate metabolite, its role in cancer development remains unknown. We found that high levels of FBP were required for cancer growth because of its ability to affect the redox status. Mechanistically, FBP regulated the redox status partially by suppressing NADPH oxidase isoform NOX4 activity in ccRCC cells. ccRCC maintained high levels of FBP through the downregulation of aldolase B (ALDOB). Reduction of FBP levels in cancer cells by the ectopic expression of ALDOB disrupted redox homeostasis, arrested cancer proliferation, and sensitized ccRCC cells to a chemotherapy agent (paclitaxel). Furthermore, low expression of ALDOB portended significantly worse disease-free survival and overall survival in ccRCC patients. In summary, the downregulation of ALDOB and accumulation of FBP promote ccRCC growth by counteracting oxidative stress.
\end{abstract}

\section{Introduction}

Cancer cells exhibit the activation of specific metabolic patterns (e.g., the Warburg effect) to provide for their energy demands. More importantly, increased steady-state concentrations of specific metabolites, such as 2-hydroxyglutarate, fumarate, and succinate, can result in altered cell signaling and promote cancer initiation and progression [1]. In our previous research, we noticed that fructose 1,6bisphosphate (FBP) levels were nearly threefold higher in clear cell renal cell carcinoma (ccRCC) tissue than those in normal kidney tissue, but the steady-state concentrations of metabolites in the earlier stages of glycolysis were

Supplementary information The online version of this article (https:// doi.org/10.1038/s41374-019-0203-3) contains supplementary material, which is available to authorized users.

Jianxin Qiu

jasonqiu@sjtu.edu.cn

1 Department of Urology, Shanghai General Hospital, School of Medicine, Shanghai Jiao Tong University, Shanghai 200080, China

2 Department of Urology, The Sixth Affiliated Hospital of Wenzhou Medical University (The People's Hospital of Lishui), Zhejiang 323000, China significantly reduced [2]. Similarly, a metabolic profile dataset from nearly 140 primary ccRCC tumors revealed the distinct behavior of metabolites in the upper and lower parts of glycolysis, suggesting that flux through glycolysis may be differentially partitioned [3]. Thus, FBP forms an interesting watershed in glycolysis. However, whether FBP is able to afford an advantage to tumor cells and its underlying mechanism remain unknown.

Previous research has shown that FBP acts as a bioactive molecule not just simply as a fuel. Statistical simulations of metabolomics data have shown that FBP is highly dynamic and variable in cancer. Simulated probability density function of FBP levels exhibited a bimodal distribution using Monte Carlo sampling [4]. Despite the allosteric regulation of pyruvate kinase, it has been found that the absence of FBP is capable of activating metabolic tumor suppressor adenosine monophosphate-activated protein kinase (AMPK) in mouse embryonic fibroblasts and HEK293 cells [5]. FBP triggers the activation of Ras in yeast [6]. FBP directly binds to the epidermal growth factor receptor and enhances the activity of the epidermal growth factor receptor in triple-negative breast cancer [7]. Our previous research has demonstrated that high levels of FBP block the activation of the p53 pathway [2].

The role of FBP in ccRCC metabolism is not clear. In this study, we confirmed the accumulation of FBP in human 
ccRCC and found that the downregulation of aldolase $\mathrm{B}$ (ALDOB) contributes to increased levels of FBP. FBP alters the redox status and preserves dihydronicotinamideadenine dinucleotide phosphate (NADPH) independent of the pentose phosphate pathway. Downregulation of ALDOB and accumulation of FBP promote ccRCC growth and development in vitro and in vivo. Furthermore, the low expression of ALDOB predicts poorer survival. Our studies identify ALDOB and FBP as critical reactive oxygen species (ROS) regulators, which contribute to tumor progression.

\section{Materials and methods}

\section{Samples}

The authors' institutional review board approved the experiments in this study. Informed consent was obtained from all the patients. Tissue samples were collected from surgical patients at Shanghai General Hospital, Shanghai Jiao Tong University School of Medicine.

\section{Cell culture}

The Caki-1 and OSRC-2 cell lines were obtained from the Type Culture Collection of the Chinese Academy of Sciences, Shanghai, China. All cells were cultured in RPMI 1640 (Invitrogen Life Technologies, USA) supplemented with $10 \%$ fetal bovine serum, $2 \mathrm{mmol} / \mathrm{l}$ L-glutamine, $100 \mathrm{U} / \mathrm{ml}$ penicillin, and $100 \mathrm{~g} / \mathrm{ml}$ streptomycin at $37^{\circ} \mathrm{C}$ in a humidified $5 \% \mathrm{CO}_{2}$ atmosphere.

\section{Immunofluorescence}

Briefly, the slices were first fixed, then washed three times with phosphate-buffered saline (PBS), and incubated with indicated primary antibodies (dilution ratio 1:100) overnight. The primary antibodies were ALDOA (Proteintech Group, USA), ALDOB (Proteintech Group, USA), and ALDOC (Sigma-Aldrich, USA). 4',6-diamidino-2-phenylindole was used for nuclear staining. All images were photographed at the same exposure time with a Nikon Eclipse Ti microscope.

\section{siRNA transfection}

Custom-designed siRNAs directed against G6PD (siG6PD1: TGATGAAGAGAGTGGGTTT; siG6PD2: AG TCGGATACACACATATT) and NOX4 (siNOX4-1: GC CUCAGCAUCUGUUCUUATT; siNOX4-2: CCAGGAG AUUGUUGGAUAATT) were synthesized and annealed. Using lipofectamine RNAiMAX (Invitrogen Life Technologies,
USA), the cells were transfected with siRNAs according to the manufacturer's instructions.

\section{cDNA transfection}

The cDNA of human ALDOB was obtained by reverse transcription polymerase chain reaction and verified by sequencing. The ALDOB cDNA was then subcloned into the lentiviral vector pCDH-CMV-EF1-Puro (System Biosciences). The ALDOB cDNA, together with the packaging plasmids PMD2G and PSPAX2, was transfected into $293 \mathrm{~T}$ cells with Lipofectamine 2000 (Invitrogen Life Technologies, USA) according to the manufacturer's instructions. Viral supernatant was collected after $48 \mathrm{~h}$. Five milliliters of viral supernatant and $5 \mathrm{ml}$ of fresh medium were added to OSRC-2 and Caki-1 cells plated in $10 \mathrm{~cm}$ dishes. Cells stably transfected with ALDOB were selected using $1 \mu \mathrm{g} / \mathrm{ml}$ puromycin.

\section{Western blot analysis}

Total protein extracts from human tissue and cells were obtained by using the radioimmunoprecipitation assay lysis buffer (Sigma, USA). Protein samples were subjected to electrophoresis by using 7.5 and $15 \%$ sodium dodecyl sulfate-polyacrylamide gel electrophoresis gels and transferred to Immuno-blot PVDF Membrane (Bio-Rad, USA). Antibodies against ALDOB (Proteintech Group, USA), ALDOA (Proteintech Group, USA), HIF1a (Cell Signaling Technology, USA), HIF2a (AbSci, USA), NOX4 (Novus, USA), $\beta$-actin (Sigma-Aldrich, USA), and $\alpha$-tubulin (Proteintech Group, USA) were used for western blotting.

\section{Cell proliferation}

Cells were replated in six-well plates with a density of 4000 cells/well. Cell numbers were counted on days 1, 2, 3, and 4 after replating.

\section{Bromodeoxyuridine assay}

For bromodeoxyuridine (BrdU) analysis, cells were pulsed with $10 \mu \mathrm{M}$ BrdU (BD Biosciences, USA) for $5 \mathrm{~h}$, then trypsinized, fixed, and labeled using BrdU Flow Kits (BD Biosciences, USA) according to the manufacturer's instructions, and analyzed by flow cytometry using a FACSCalibur flow cytometer (BD Biosciences, USA).

\section{Oxidative DNA damage quantification assay}

DNA damage was assessed by the quantification of apurinic/apyrimidinic (AP) sites using the DNA damage 
quantification kit (BioVision, USA) according to the manufacturer's instructions.

\section{ROS measurement}

Cellular ROS content was determined by using the dichlorofluorescein (DCFH) diacetate probe according to the manufacturer's instructions (Sigma-Aldrich). Briefly, cells were incubated with $10 \mu \mathrm{M} \mathrm{DCFH}$ at $37^{\circ} \mathrm{C}$ for $10 \mathrm{~min}$ in the dark and washed with PBS. Washed cells were then trypsinized and suspended for flow cytometry. Alternatively, following the treatment, the medium was removed and cells were loaded with $10 \mu \mathrm{M}$ DCFH diluted in PBS for $15 \mathrm{~min}$ at $37^{\circ} \mathrm{C}$. Cells were washed three times with PBS, and fluorescence intensity was measured using a plate reader. For the measurement of mitochondrial ROS, cells cultured in 96-well plates were washed with PBS, incubated with $4 \mu \mathrm{M}$ MitoSOX (Invitrogen, USA) in media for about $15 \mathrm{~min}$, cells washed again with PBS, and then the plates were read in the plate reader.

\section{Metabolic assays}

Enzymatic measurements of FBP were conducted according to the described procedure [2]. Briefly, the neutralized extract was added in the reaction buffer that contains 0.5 $\mathrm{mM}$ Na-ethylenediaminetetraacetic acid, $50 \mathrm{mM}$ Tris- $\mathrm{HCl}$ (pH 7.5), and 0.2 mM NADH. Sequential addition of $0.2 \mathrm{U}$ glycerol-3-phosphate dehydrogenase (Sigma, USA), $1 \mathrm{U}$ triosephosphate isomerase (Sigma, USA), and $0.2 \mathrm{U}$ aldolase (Sigma, USA) catalyzes the reactions. Absorbance was recorded at $340 \mathrm{~nm}$ at room temperature by using a spectrophotometer.

\section{Metabolomic quantitation by high-performance liquid chromatography-mass spectrometry}

For metabolite extraction, cells were washed three times using PBS and harvested in a solution of acetonitrile, methanol, and water (2/2/1 by v/v; all HPLC grade). These extracts were centrifuged at $12000 \times \mathrm{g}$ for $10 \mathrm{~min}$ at $4{ }^{\circ} \mathrm{C}$, and the supernatants were dried under $\mathrm{N}_{2}$ stream at room temperature. Liquid chromatography-tandem mass spectrometry data were acquired by following the previously described methods [2].

\section{NADPH oxidase assay}

NADPH-dependent superoxide generation in cell extracts was measured by lucigenin-enhanced chemiluminescence as previously described $[8,9]$.

\section{Animal experiments}

All animal experiments were conducted following protocols approved by the Institutional Animal Care and Use Committee. Female BALB/c nude mice were ordered from the Shanghai SLAC Laboratory Animal Co. Ltd. and subjected to subcutaneous tumorigenesis as described previously [2]. Briefly, $1 \times 10^{7}$ indicated cells were resuspended in $200 \mu \mathrm{l}$ PBS and subcutaneously injected into the flanks of nude mice. Intraperitoneal paclitaxel, $10 \mathrm{mg} / \mathrm{kg}$, on day 14,18 , and 22 schedules, was administered to mice bearing subcutaneous xenografts. Four weeks after injection, tumors were excised, weighed, and assayed for protein expression.

\section{RNA sequencing data analysis}

Raw sequence files of GSE102101 and GSE76207 datasets were downloaded from sites of GEO [10]. Reads were aligned to the human GRCh38 reference genome using HISAT (2.1.0) [11] and counted by using featureCounts (1.6.2) [12].

\section{Statistical analysis}

Statistical analysis was performed by using $\mathrm{R}$ version 3.2.3 (http://www.r-project.org/). Experiments were performed at least three times independently. $P$ values were two-sided; it was considered statistically significant when $P<0.05$.

\section{Results}

\section{FBP accumulates in cCRCC and controls redox status}

Previous studies have shown the metabolic profiling in normal kidney tissue and ccRCC tissue by using liquid chromatography-mass spectrometry (LCMS) [3]. Compared with normal kidney tissue, the isobars including FBP, glucose-1,6-bisphosphate, myo-inositol-1,4, and 1,3-bisphosphate were upregulated in ccRCC tissue. To confirm the accumulation of FBP in ccRCC tissue, we used enzymatic methods to detect FBP, dihydroxyacetone phosphate (DHAP), and glyceraldehyde 3-phosphate (G3P) without the interference of isobars. In line with the LCMS results, levels of FBP in ccRCC were significantly higher than those of normal kidney tissue, while the products of aldolase, DHAP, and G3P were significantly downregulated in ccRCC compared with normal kidney tissue (Fig. 1a). Analysis of previously reported LCMS dataset also showed that the abundances of metabolites of the lower part of glycolysis, such as 3-phosphoglyceric acid, 2-phosphoglyceric acid (2PG), and phosphoenolpyruvate, were interestingly lower in ccRCC tissue (Supplementary Fig. S1) [3]. 

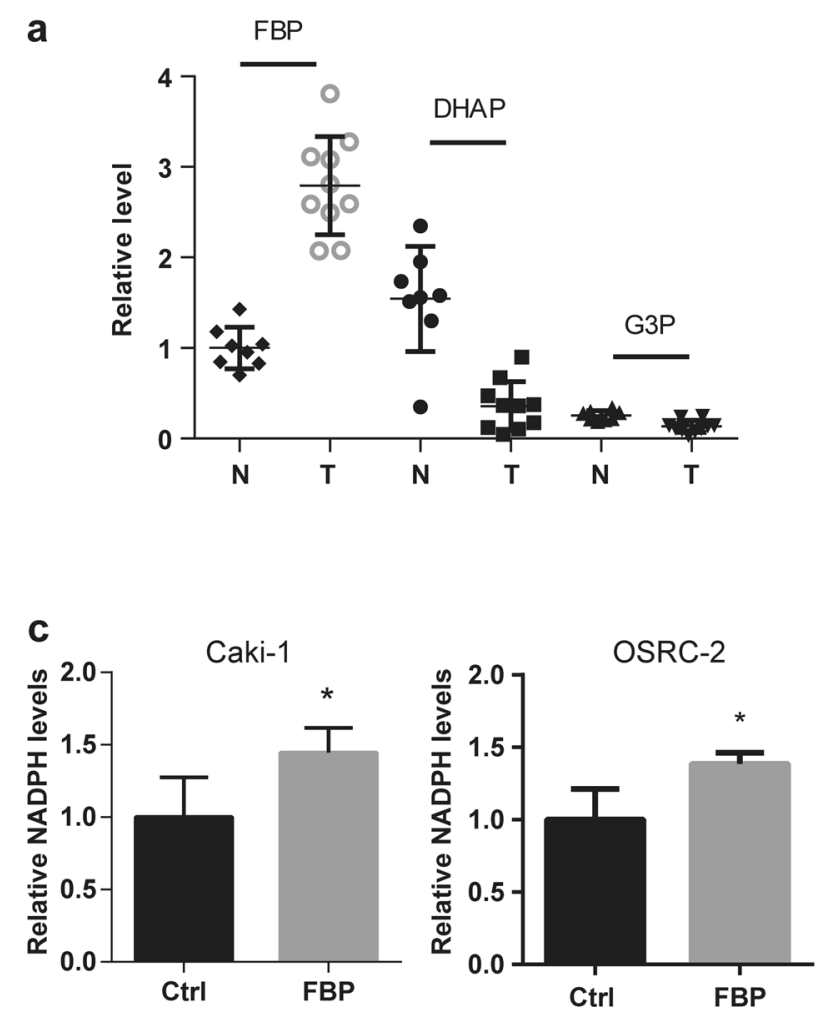

Fig. 1 FBP accumulates in ccRCC and controls redox status. a Relative concentrations of FBP, DHAP, and G3P in 10 human RCC tissue samples (T) and 8 adjacent non-malignant kidney tissue samples $(\mathrm{N})$ measured using an enzymatic approach. b LCMS profile of Caki-1 cells incubated with FBP compared with control cells. c Relative NADPH levels of Caki-1 and OSRC-2 cells treated with $1 \mathrm{mM} \mathrm{FBP.}$

We first hypothesized that the accumulation of FBP might affect HIF1a and HIF2a in ccRCC in a manner similar to the effects of fumarate in fumarate hydratasedeficient renal cancers. However, FBP treatment did not significantly alter the levels of HIF1a and HIF2a (Supplementary Fig. S2). We then evaluated the significance of FBP accumulation in cancer metabolism. We incubated Caki-1 cells with FBP, and intracellular metabolites were detected by using LCMS (Fig. 1b). The rise of intracellular FBP only led to a slight accumulation of fructose 6phosphate (F6P) and DHAP/G3P. Levels of FBP did not significantly influence the product of the pentose phosphate pathway (ribulose-5-phosphate) and the lower part of glycolysis (i.e., 3-phosphoglyceric acid, 2-phosphoglyceric acid) (Fig. 1b). More interestingly, FBP conserved NADPH and altered the redox status in Caki-1 and OSRC-2 cells (Fig. 1c, d).

\section{FBP regulates redox status partially by suppressing the activity of NOX4}

Then, we explored the mechanism of FBP affecting ROS. To investigate whether FBP altered the redox status through the b
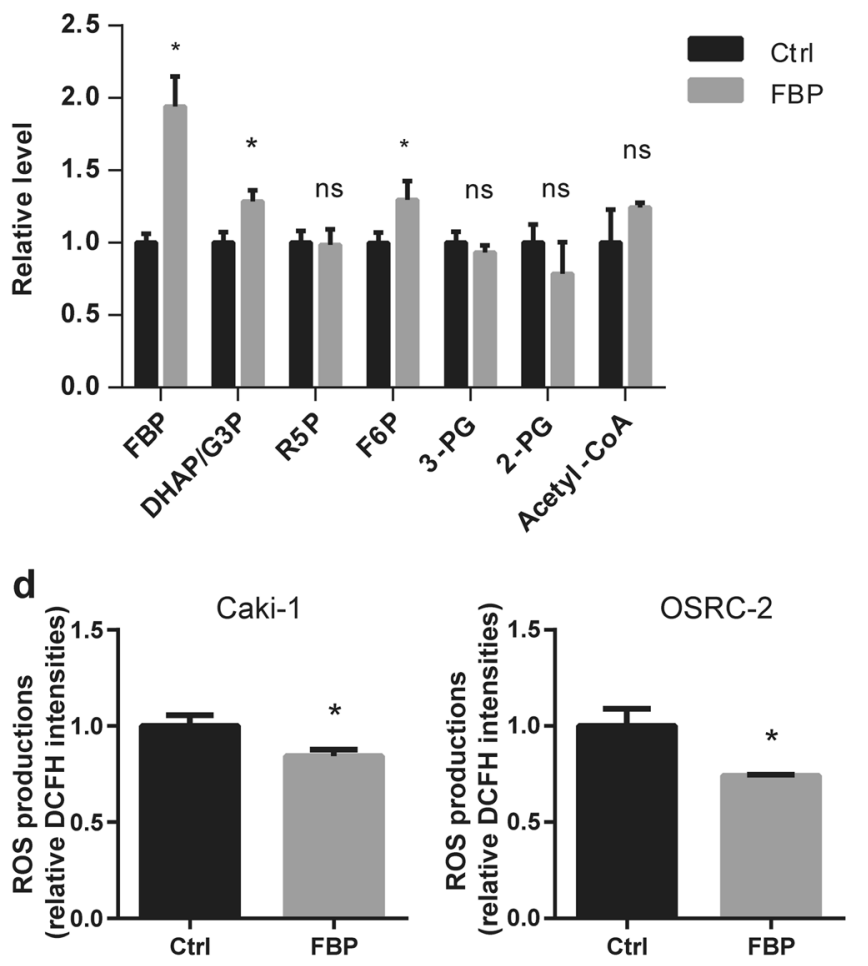

d Relative DCFH intensities of Caki-1 and OSRC-2 cells treated with $1 \mathrm{mM}$ FBP. ccRCC, clear cell renal cell carcinoma; DCFH, dichlorofluorescein; DHAP, dihydroxyacetone phosphate; FBP, fructose 1,6bisphosphate; G3P, glyceraldehyde 3-phosphate; LCMS, liquid chromatography-mass spectrometry

oxidation branch of the pentose phosphate pathway, we knocked down glucose-6-phosphate dehydrogenase (G6PD), which is the key enzyme in the oxidation branch of the pentose phosphate pathway (Fig. 2a). G6PD depletion did not abolish the effect of FBP (Fig. 2b, c), which indicates that FBP's effect is independent of the pentose phosphate pathway. Further, to investigate whether the effects of FBP were through the effects of upstream or downstream metabolites (F6P, G3P, and DHAP), we incubated cells with these metabolites $(1 \mathrm{mM})$. A supplement of these intermediate metabolites did not significantly change the redox status (data not shown).

We detected mitochondrial ROS with mitochondriaspecific probe MitoSOX. Consistent with the previous total ROS results, FBP also reduced mitochondrial ROS levels (Fig. 2d). Kidney-specific NADPH oxidase isoform 4 (NOX4) also produces considerable amounts of ROS in ccRCC $[8,9,13]$. To determine whether FBP regulates the activities of NADPH oxidases, activities of NADPH oxidases in Caki-1 cells were measured. Addition of FBP (200 $\mu \mathrm{M})$ into Caki-1 cell extracts significantly decreased the activities of total NADPH oxidases, suggesting that NADPH oxidases may be involved (Fig. 2e). As NOX4 is the 
a

siCtrl siG6PD1 siCtrl siG6PD2

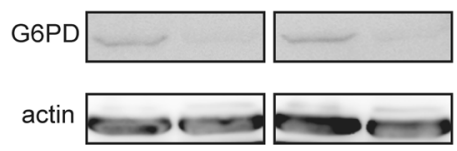

e

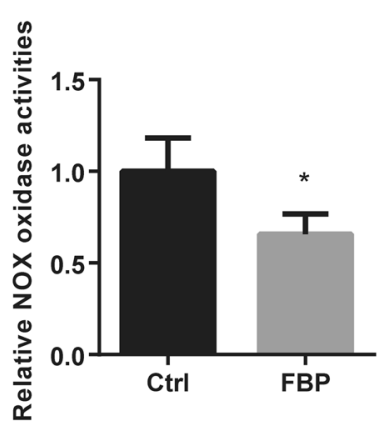

b

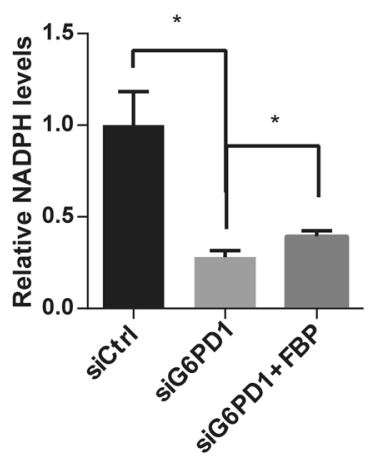

f

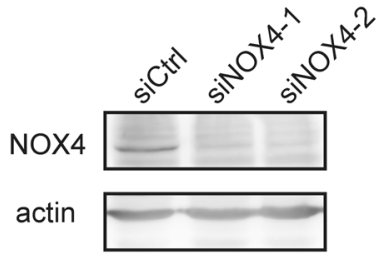

C
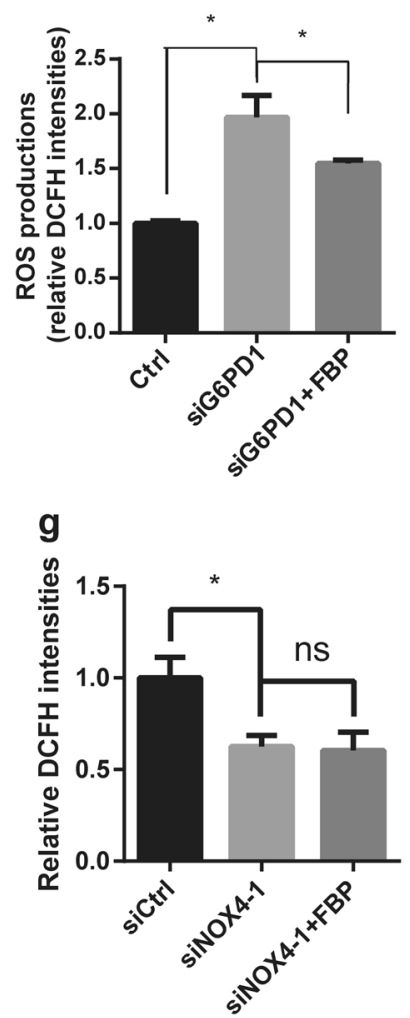

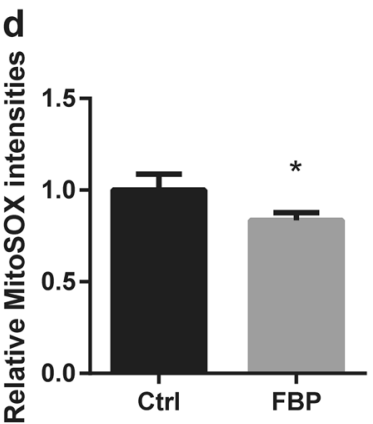

Fig. 2 FBP regulates redox status partially by suppressing the activity of NOX4. a Western blot analysis of Caki-1 cells transiently transfected with siCtrl, siG6PD1, or siG6PD2 at $72 \mathrm{~h}$ after transfection. b Relative NADPH levels of Caki-1 cells transiently transfected with siCtrl, siG6PD1, and siG6PD1 followed by $1 \mathrm{mM}$ FBP incubation at $72 \mathrm{~h}$ after transfection. c Relative DCFH intensities of Caki-1 cells transiently transfected with siCtrl, siG6PD1, and siG6PD1 followed by $1 \mathrm{mM}$ FBP incubation at $72 \mathrm{~h}$ after transfection. d Relative MitoSOX

predominant isoform in ccRCC [8], we knocked down NOX4 in Caki-1 cells (Fig. 2f). Compared with control cells, NOX4 knockdown significantly reduced the ROS levels in ccRCC cells and partially abolished the impact of FBP on redox status, suggesting that FBP regulates redox status partially by suppressing the activity of NOX4 (Fig. 2g).

\section{Downregulation of ALDOB contributes to FBP accumulation}

A change in the level of an individual metabolite is caused by a change in the rate of production relative to the rate of consumption in a metabolic pathway. FBP is mainly formed by phosphofructokinase-1 (PFK1) and metabolized by aldolase. Our previous study had shown that PFK1 platelet isoform was upregulated in ccRCC and its depletion led to decreased levels of FBP. But it does not explain the shrinkage of the lower part of glycolysis. In humans, there are three isoenzymes (ALDOA, ALDOB, and ALDOC) in the aldolase family. We analyzed two RNA sequencing intensities of Caki-1 treated with $1 \mathrm{mM}$ FBP. e Relative NADPH oxidase activities (relative luminometer units) of Caki-1 cell extracts treated with or without FBP. f Western blot analysis of Caki-1 cells transiently transfected with siCtrl, siNOX4-1, or siNOX4-2 at $72 \mathrm{~h}$ after transfection. g Relative DCFH intensities of Caki-1 cells transiently transfected with siCtrl, siNOX4-1, and siNOX4-1 followed by $1 \mathrm{mM}$ FBP incubation at $72 \mathrm{~h}$ after transfection. $* P<0.05$. DCFH, dichlorofluorescein diacetate; FBP, fructose 1,6-bisphosphate

datasets from GEO (gene expression omnibus) and found that ALDOB was the predominant form in the context of kidney and almost diminished in ccRCC (Fig. 3a, b). ALDOC was barely detectable in both normal kidney and ccRCC tissue. Although the levels of ALDOA were slightly higher in ccRCC, the total abundance of aldolase diminished in ccRCC. Consistent with the results of RNA sequencing analysis, immunofluorescence showed that ALDOB was depleted in ccRCC tissue; ALDOC was almost undetectable in normal and tumor slices (Fig. 3c). Western blotting further confirmed that ALDOB was significantly downregulated in ccRCC; ALDOA was at a similar level compared with normal kidney tissue (Fig. 3d). We compared fold changes of ALDOB among cancer types in The Cancer Genome Atlas (TCGA) program (types with normal tissue sample size $<10$ were excluded) and found that ccRCC and two relatively rare subtypes of kidney cancer had the three highest fold changes of downregulation of ALDOB, which indicates that downregulation of ALDOB may play an important role in kidney cancer (Fig. 3e). 
a



b

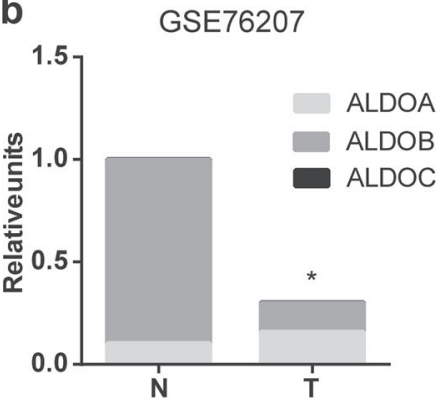

d
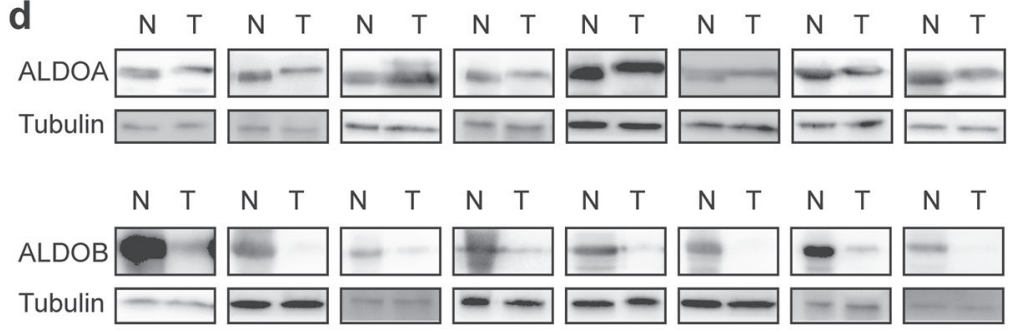

e

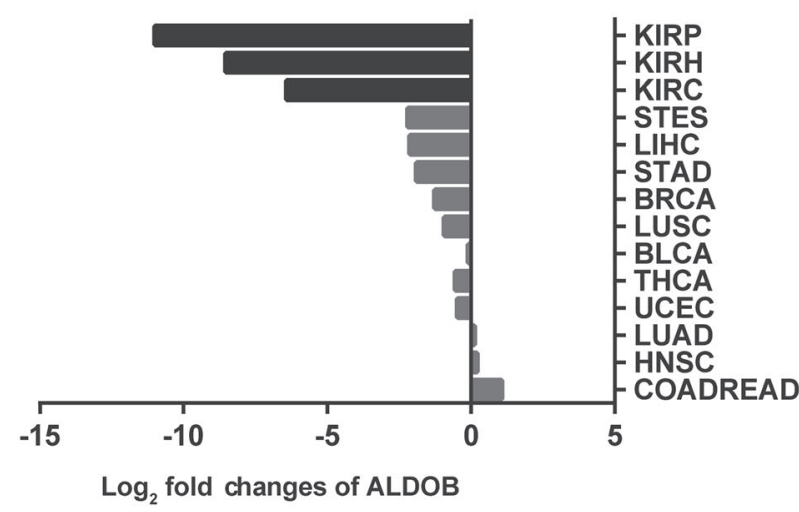

c

$\mathrm{N}$
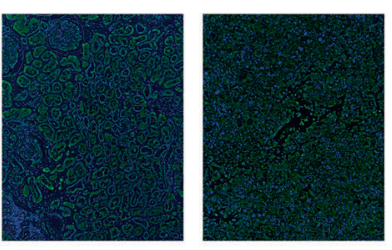

ALDOA
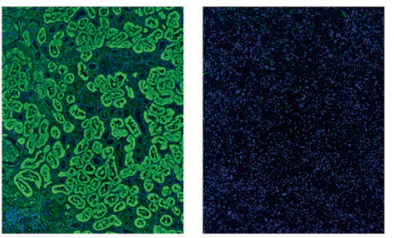

ALDOB
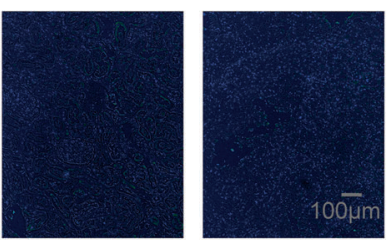

f

Caki-1 OSRC-2

Ctrl ALDOB Ctrl ALDOB

ALDOB

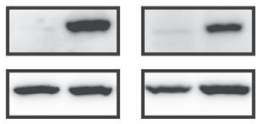

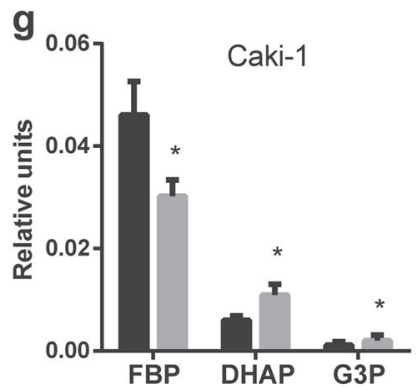

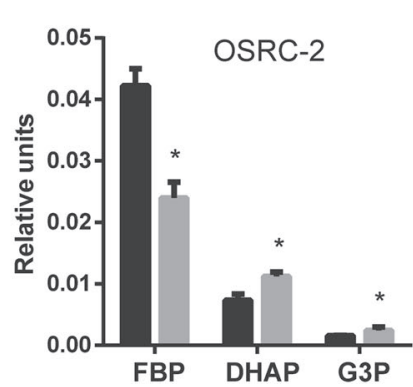

Fig. 3 Downregulation of ALDOB contributes to FBP accumulation. a Mean abundance of ALDOA, ALDOB, and ALDOC from dataset (GSE102101) in ccRCC and normal kidney tissue $(N=20)$. b Mean abundance of ALDOA, ALDOB, and ALDOC from dataset (GSE76207) in ccRCC and normal kidney tissue $(N=32)$. c The tissue slices of the human kidney $(\mathrm{N})$ and ccRCC $(\mathrm{T})$ were incubated with indicated antibodies. DAPI was used to achieve nuclear staining. All images were acquired under the same conditions and displayed at the same scale. Magnification: $10 \times$. d Western blot analysis of ALDOA and ALDOB for eight paired ccRCC and normal kidney tissue. e Fold changes of mean abundance of ALDOB from TCGA in various cohorts: KIRP (kidney renal papillary cell carcinoma), $\mathrm{KICH}$ (kidney chromophobe), KIRC (kidney renal clear cell carcinoma), STES (stomach and esophageal carcinoma), LIHC (liver hepatocellular carcinoma), STAD (stomach adenocarcinoma), BRCA (breast
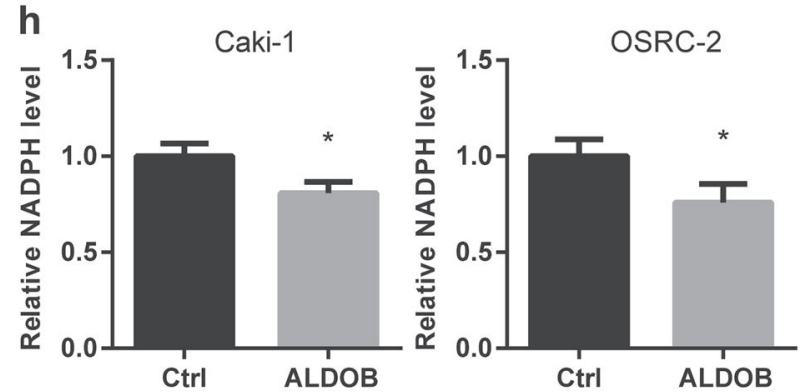

invasive carcinoma), LUSC (lung squamous cell carcinoma), BLCA (bladder urothelial carcinoma), THCA (thyroid carcinoma), UCEC (uterine corpus endometrial carcinoma), LUAD (lung adenocarcinoma), HNSC (head and neck squamous cell carcinoma), and COADREAD (colon adenocarcinoma and rectum adenocarcinoma). $\mathbf{f}$ Western blot analysis of Caki-1 and OSRC-2 cells stably transfected with control vector (Ctrl) or ALDOB. g Enzymatic quantification of FBP, DHAP, and G3P in Caki-1 and OSRC-2 cells stably and ectopically expressing ALDOB compared with control cells. h Relative NADPH levels of Caki- 1 and OSRC- 2 cells stably and ectopically expressing ALDOB compared with control cells. $* P<0.05$. ALDOB, aldolase B; ccRCC, clear cell renal cell carcinoma; DCFH, dichlorofluorescein; DHAP, dihydroxyacetone phosphate; FBP, fructose 1,6bisphosphate; G3P, glyceraldehyde 3-phosphate; TCGA, The Cancer Genome Atlas 
FBP accumulation, we established two ccRCC cell lines that stably expressed ALDOB: Caki-1-ALDOB and OSRC2-ALDOB (Fig. 3f). ALDOB overexpression decreased the levels of FBP and increased the levels of DHAP and G3P (Fig. 3g). ALDOB overexpression decreased NADPH levels in Caki-1-ALDOB and OSRC-2-ALDOB cells compared with control cells (Fig. 3h).

\section{Downregulation of ALDOB and accumulation of FBP counteract oxidative stress to support cCRCC development}

We postulated that the accumulation of FBP in cancer would substantially affect the metabolism of cancer cells. As cancer cells already have relatively high levels of FBP, we used Caki-1-ALDOB and OSRC-2-ALDOB as models for lowFBP cells. Ectopic expression of ALDOB alone only had limited effects on the proliferation of Caki-1 and OSRC-2 cells (Fig. 4a, b). We next examined the effect of ALDOB on cellular capacity to withstand oxidative stresses. We used a low concentration $(10 \mu \mathrm{M})$ of hydrogen peroxide $\left(\mathrm{H}_{2} \mathrm{O}_{2}\right)$ to induce oxidative stresses without significant apoptosis. Compared to the control groups, Caki-1-ALDOB and OSRC2-ALDOB cells were much more sensitive to the low concentration $(10 \mu \mathrm{M})$ of $\mathrm{H}_{2} \mathrm{O}_{2}$ treatment. BrdU assay also revealed the decreased proliferation in Caki-1-ALDOB cells compared with negative controls under oxidative stress (Fig. 4c, d). To ascertain that the vulnerability of ALDOBexpressing cells to oxidative stresses is due to the reduction in FBP level, we tested the effect of FBP supplement in these cells. Supplement of FBP significantly enhanced the proliferation of ALDOB-expressing cells in the presence of $\mathrm{H}_{2} \mathrm{O}_{2}$ (Fig. 4a-d). Furthermore, FBP treatment inhibited oxidative DNA damage in Caki-1-ALDOB cells (Fig. 4e). These data suggest that the downregulation of ALDOB and accumulation of FBP play a role in protecting against oxidative stress.

ccRCC is typically resistant to both chemotherapy and radiotherapy. Increasing evidence has suggested that the reduction of oxidative stress protects cancer cells from chemotherapy. Several studies have utilized various means to efficiently enhance the sensitivity of ccRCC cells to chemotherapy drug paclitaxel [14-17]. In light of this, we explored if re-expression of ALDOB would sensitize cancer cells to paclitaxel treatment. We implanted Ctrl and ALDOB ectopic-expressing Caki-1 cells into nude mice, which were administered with low doses of paclitaxel. Expression of ALDOB apparently retarded the growth of tumors compared with control cells (Fig. 4f-h).

\section{Low expression of ALDOB predicts poor prognosis}

We analyzed ALDOB expression and the clinicopathological features of ccRCC tissues from the TCGA database. We noticed that the expression of ALDOB in pT2 ccRCC was significantly lower than that in pT1; the expression of ALDOB in pT4 ccRCC was significantly lower than that in pT3 (Fig. 5a). ALDOB expression was negatively correlated with distant metastasis (M1) but not with the presence of positive regional lymph nodes (Fig. 5b, c). Moreover, the expression of ALDOB in grade $4 \mathrm{ccRCC}$ was significantly lower than that in lower grades $(1,2$, and 3) (Fig. 5d). In general, our data show the downregulation of ALDOB expression in higher stage and higher grade ccRCC. We further explored whether the downregulation of ALDOB was correlated with the prognosis of patients with ccRCC. Kaplan-Meier analysis with TCGA datasets was used to compare the subgroups with low and high expression of ALDOB. Median expression levels of ALDOB were used as the cut-off point (Fig. 5e, f). Patients with low expression of ALDOB had significantly worse disease-free survival (DFS) and overall survival (OS) $(P=1.800 \mathrm{e}-5$ and $P=5.421 \mathrm{e}-5$, respectively).

\section{Discussion}

In the past decade, tremendous efforts have been made to explore the mechanisms underlying Warburg effect. There is evidence to suggest that aerobic glycolysis maximizes the production of macromolecules and antioxidants. Historically, glycolysis is thought to be a simple sequential model with three rate-limiting steps. In fact, oncogene-driven metabolic rewiring profoundly modifies the normal pathway and affects non-rate-controlling enzymes, such as glucose6-phosphate isomerase and triosephosphate isomerase 1; these enzymes also play pivotal roles in cancer progression $[18,19]$. The accumulation of fumarate activates oncogenic hypoxia-inducible factor pathways in a rare subtype of kidney cancer, HLRCC (hereditary leiomyomatosis and renal cell cancer). Our studies provide evidence that intermediate products such as FBP accumulated in ccRCC cells. Furthermore, the accumulation of FBP offers ccRCC cells additional survival advantages, especially under oxidative stress. Changes in levels of individual metabolites are caused by changes of production and consumption in metabolic pathways. Li, et al. [20] and our previous research [2] have shown that depleted fructose 1,6-bisphosphatase 1 and overexpressed PFK1 isoenzyme contribute to increasing supplies of FBP. However, low abundances of metabolites in the earlier steps of glycolysis cause us to question whether aldolase forms an alternative bottleneck in the context of rewired aerobic glycolysis. In this study, we found that ALDOB was downregulated in ccRCC; ectopic expression of ALDOB significantly reduced the difference in concentration between intracellular FBP and downstream metabolites (G3P and DHAP). In summary, the 

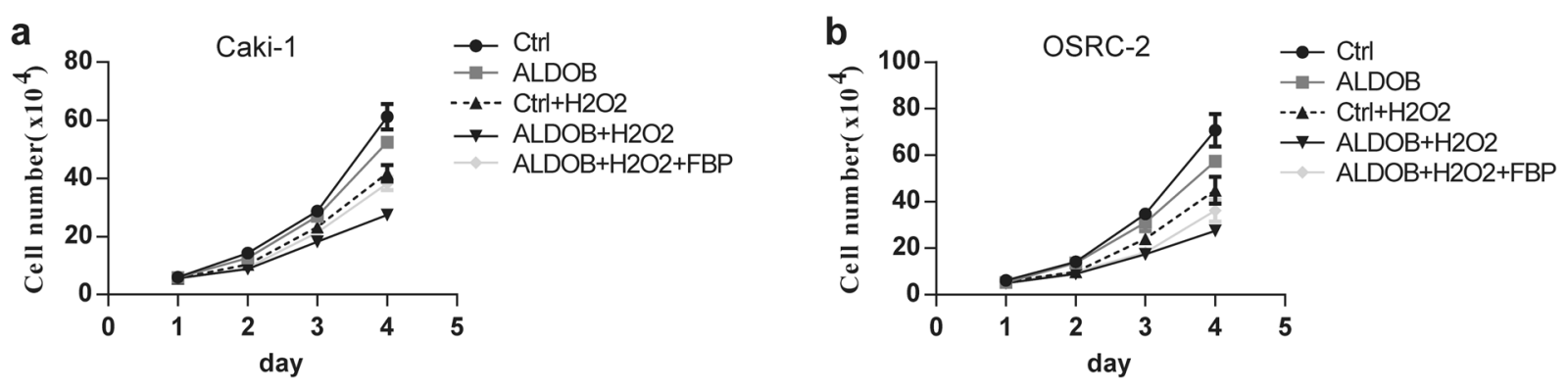

C

Ctrl

$\mathrm{Ctrl}+\mathrm{H}_{2} \mathrm{O}_{2}$

$\mathrm{ALDOB}+\mathrm{H}_{2} \mathrm{O}_{2}$

$\mathrm{ALDOB}+\mathrm{H}_{2} \mathrm{O}_{2}+\mathrm{FBP}$
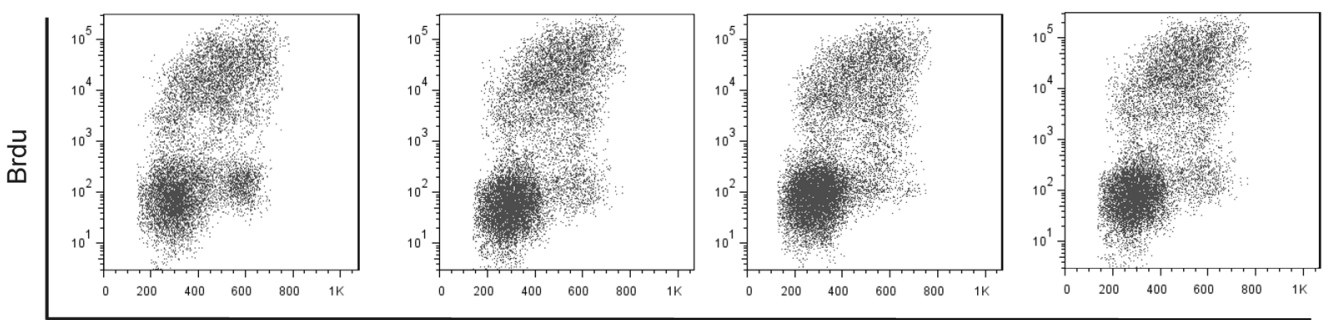

DNA content

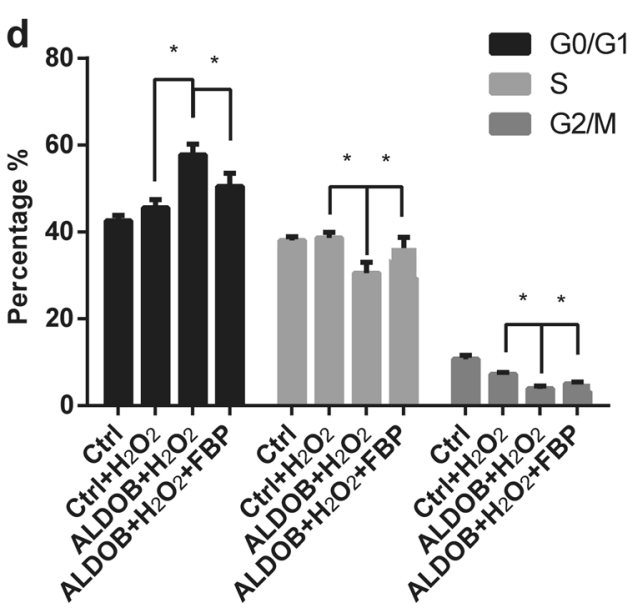

e

f
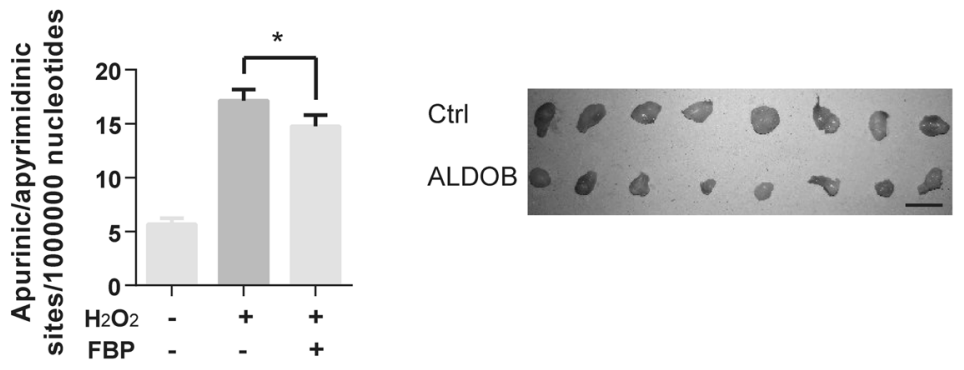

g

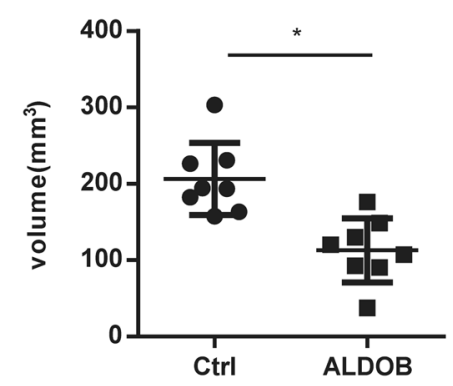

h

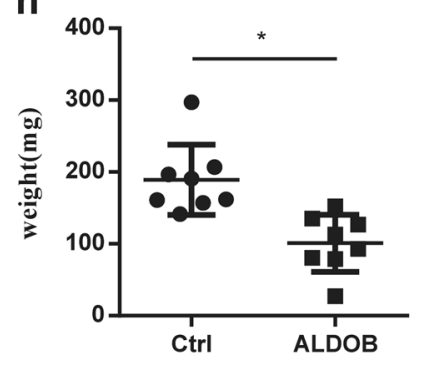

Fig. 4 Downregulation of ALDOB and accumulation of FBP counteract oxidative stress to support ccRCC development. a, b Cell growth curves of Caki-1 (a) and OSRC-2 (b) cells stably transfected with $\mathrm{Ctrl}$, ALDOB after $1 \mathrm{mM}$ FBP treatment and subjected to various treatments $\left(\mathrm{H}_{2} \mathrm{O}_{2}\right.$, FBP or a sequential incubation of $\mathrm{H}_{2} \mathrm{O}_{2}$ and FBP). c Caki-1 cells stably transfected with Ctrl or ALDOB were subjected to various treatments $\left(\mathrm{H}_{2} \mathrm{O}_{2}\right.$ or a sequential incubation of $\mathrm{H}_{2} \mathrm{O}_{2}$ and FBP) and BrdU-incorporation assay. $\mathbf{d}$ Histogram of the quantification of BrdU-incorporation assay. e Caki-1 cells stably transfected with ALDOB were subjected to $0.1 \mathrm{mM} \mathrm{H}_{2} \mathrm{O}_{2}$ or sequential incubation of
$0.1 \mathrm{mM} \mathrm{H} \mathrm{H}_{2} \mathrm{O}_{2}$ and $1 \mathrm{mM}$ FBP and quantification of apurinic/apyrimidinic (AP) sites. $\mathbf{f}$ Picture of subcutaneous tumors from nude mice subcutaneously injected with Caki-1 cells stably transfected with Ctrl or ALDOB. Scale bar $=1 \mathrm{~cm}$. g, h Weight (g) and size (h) of subcutaneous tumors from nude mice subcutaneously injected with Caki-1 cells stably transfected with Ctrl or ALDOB. $* P<0.05$. ALDOB, aldolase $\mathrm{B}$; BrdU, bromodeoxyuridine; ccRCC, clear cell renal cell carcinoma; FBP, fructose 1,6-bisphosphate; G3P, glyceraldehyde 3phosphate 

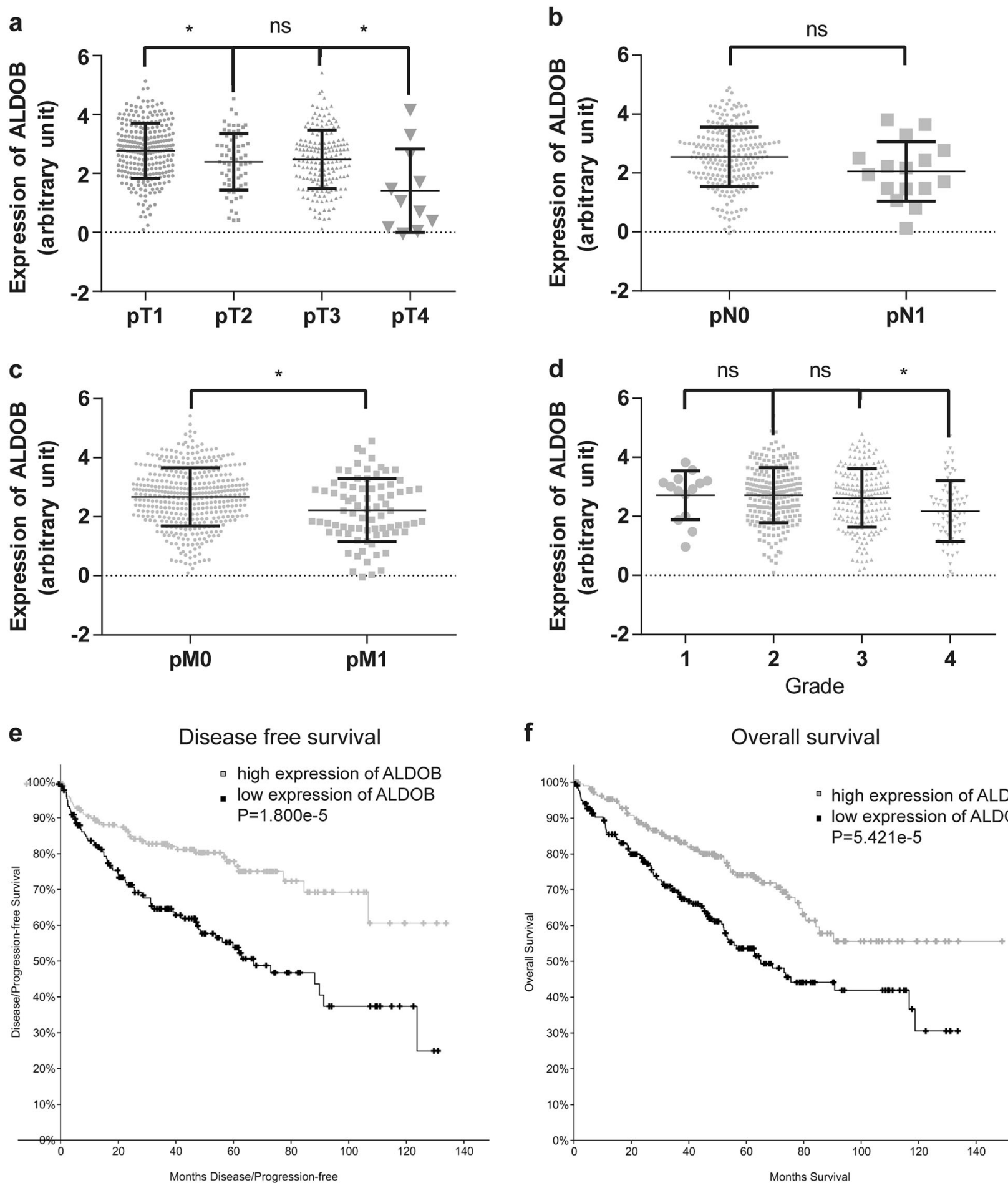

f
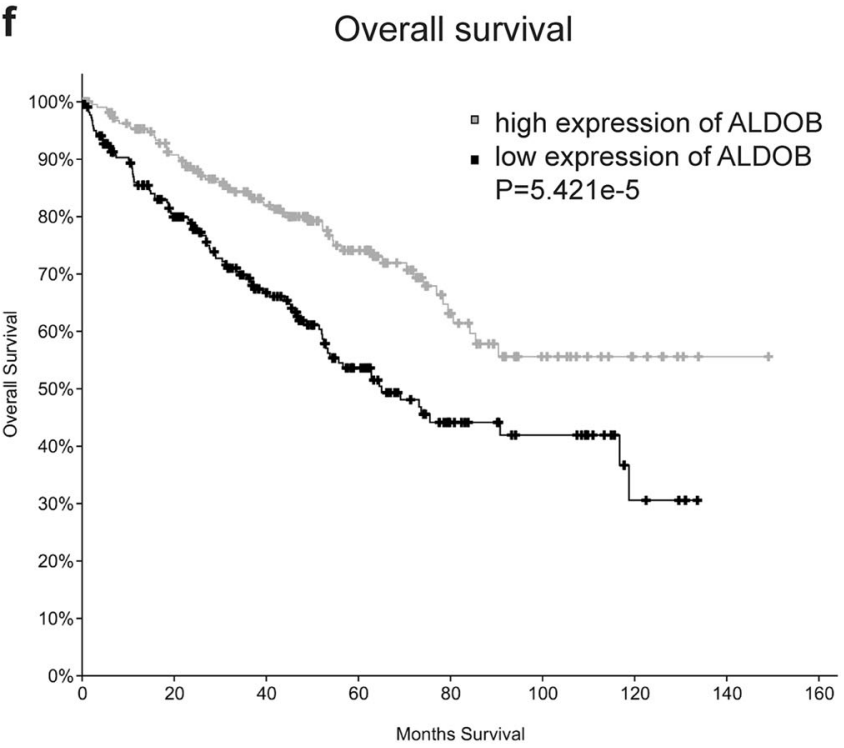

Fig. 5 Low expression of ALDOB predicts poor prognosis. a The correlation between the expression of ALDOB in ccRCC tumors and different pT (1, 2, 3, and 4). b The correlation between the expression of ALDOB in ccRCC tumors and different $\mathrm{pN}$ (0 and 1). c The correlation between the expression of ALDOB in ccRCC tumors and

downregulation of ALDOB contributes to the accumulation of FBP, which counteracts oxidative stress to support ccRCC development (Fig. 6).

different $\mathrm{pM}(0$ and 1$)$. d The correlation between the expression of ALDOB in ccRCC tumors and different grades (1, 2, 3, and 4). e, f Kaplan-Meier curves for disease-free survival (e) and overall survival (f) in RCC patients with low and high ALDOB expression. $* P<0.05$. ALDOB, aldolase B; ccRCC, clear cell renal cell carcinoma

Mechanistically, we found that FBP regulates ROS levels partially by suppressing the activities of NOX4 in ccRCC (Fig. 6). In contrast to other NOX isoforms, NOX4 


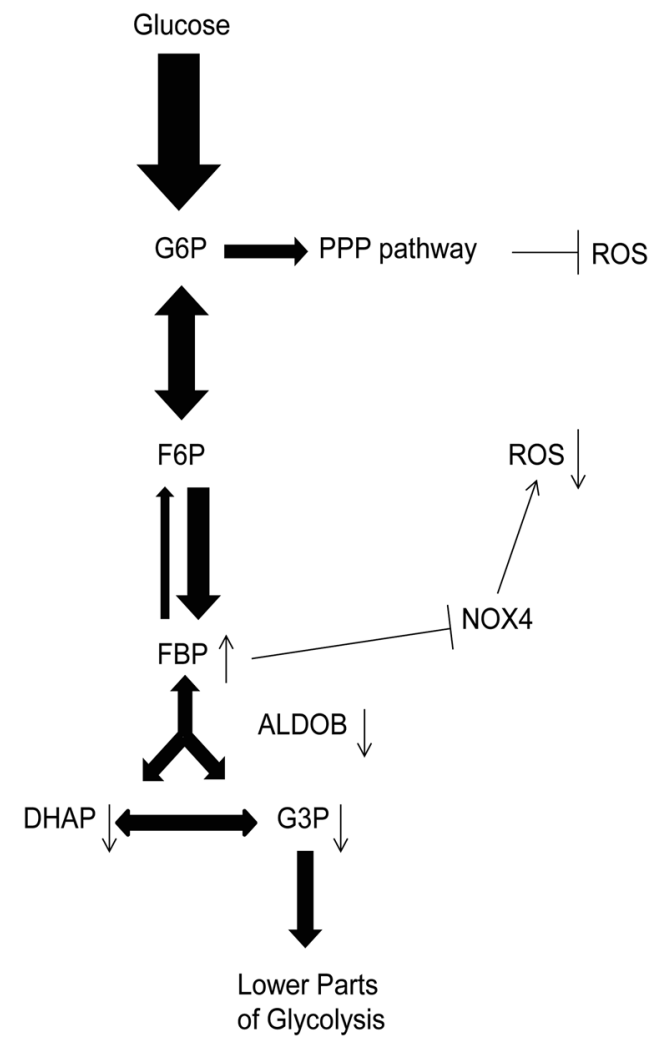

Fig. 6 a Schematic diagram of a proposed mechanism for the protective role of FBP against oxidative stress in ccRCC. Downregulation of ALDOB and accumulation of FBP protect ccRCC from oxidative stress partially by the inhibition of NOX4 activities. The / symbol indicates activation or induction and $\perp$ indicates inhibition or blockade. Summary 18-0679-AR. Clear cell renal cell carcinoma (ccRCC) is characterized by the activation of hypoxia-inducible factors and enhanced aerobic glycolysis. In this study, the authors found that ccRCC maintains high levels of fructose 1,6-bisphosphate (FBP) through the downregulation of aldolase B. In turn, FBP regulates the redox status of the tumor by suppressing NADPH oxidase isoform NOX4 activity. Thus, aldolase B and FBP are critical regulators of reactive oxygen species and contribute to tumor progression

is the only NADPH oxidase highly expressed in ccRCC cells [8]. Apart from FBP, NOX4 is also negatively regulated by another high-energy molecule, ATP [9]. Additionally, NOX4 is closely associated with the HIF2a pathway in ccRCC [13]. In fact, FBP suppresses ROS production through multiple mechanisms. Earlier studies have shown that FBP protects the brain from ischemia-reperfusion injury [21]; FBP has also been clinically used as a neuroprotective agent to treat stroke for decades. Several mechanisms have been postulated for the effects of FBP, including the repression of mitochondrial oxidative phosphorylation, direct chelation of calcium, and sequestration of ferrous to inhibit Fenton-related reactivity [22-24]. Furthermore, FBP alters intracellular nitric oxide production and inhibits the conversion of xanthine dehydrogenase to xanthine oxidase [24]. These mechanisms are able to lower the production of ROS. These may be the reasons that kidney cancer cells accumulate FBP and survive under exogenous oxidative stress. In addition, the downregulation of ALDOB affords an advantage to cancer cells by lowering the production of toxic methylglyoxal, a product formed by the spontaneous decomposition of G3P and DHAP. We also found that ALDOB expression was negatively correlated with the prognosis of ccRCC patients. However, the prognostic value of FBP remains unknown. This is one of the potential weaknesses of this study.

In humans, there are three isoenzymes in the aldolase family: ALDOA, which is predominantly found in the muscle tissue and erythrocytes; ALDOB, in the liver, kidney, and intestine; and ALDOC, in the central nervous system. ALDOB also plays a pivotal role in fructose metabolism. Defects in ALDOB can cause the accumulation of fructose 1-phosphate and hereditary fructose intolerance. We observed a ninefold increase in fructose 1-phosphate accumulation in ccRCC. More interestingly, since the liver is the predominant organ to metabolize fructose, hepatocellular carcinoma also significantly downregulates ALDOB [25], which theoretically blocks the metabolism of fructose. Somehow, it seems contradictory to some reports that declared the importance of fructose utilization in acute myeloid leukemia and pancreatic cancer [26, 27]. Thus, further research is needed.

Acknowledgements This study was supported by the Natural Science Foundation from Science and Technology Commission of Shanghai (grant number 134119a2801).

\section{Compliance with ethical standards}

Conflict of interest The authors declare that they have no conflict of interest.

Publisher's note: Springer Nature remains neutral with regard to jurisdictional claims in published maps and institutional affiliations.

\section{References}

1. Sullivan LB, Gui DY, Heiden MGV. Altered metabolite levels in cancer: implications for tumour biology and cancer therapy. Nat Rev Cancer. 2016;16:680.

2. Wang J, Zhang P, Zhong J, Tan M, Ge J, Tao L, et al. The platelet isoform of phosphofructokinase contributes to metabolic reprogramming and maintains cell proliferation in clear cell renal cell carcinoma. Oncotarget. 2016;7:27142-57.

3. Hakimi AA, Reznik E, Lee C-H, Creighton CJ, Brannon AR, Luna A, et al. An integrated metabolic atlas of clear cell renal cell carcinoma. Cancer Cell. 2016;29:104-16.

4. Shestov AA, Liu X, Ser Z, Cluntun AA, Hung YP, Huang L, et al. Quantitative determinants of aerobic glycolysis identify flux through the enzyme GAPDH as a limiting step. eLife. 2014;3: $\mathrm{e} 03342$. 
5. Zhang CS, Hawley SA, Zong Y, Li M, Wang Z, Gray A, et al. Fructose-1,6-bisphosphate and aldolase mediate glucose sensing by AMPK. Nature. 2017;548:112-6.

6. Peeters K, Van Leemputte F, Fischer B, Bonini BM, Quezada H, Tsytlonok M, et al. Fructose-1,6-bisphosphate couples glycolytic flux to activation of Ras. Nat Commun. 2017;8:922.

7. Lim S-O, Li C-W, Xia W, Lee H-H, Chang S-S, Shen J, et al. EGFR signaling enhances aerobic glycolysis in triple negative breast cancer cells to promote tumor growth and immune escape. Cancer Res. 2016;76:1284-96.

8. Gregg JL, Turner RM, Chang G, Joshi D, Zhan Y, Chen L, et al. NADPH oxidase NOX4 supports renal tumorigenesis by promoting the expression and nuclear accumulation of HIF2 $\alpha$. Cancer Res. 2014;74:3501-11.

9. Shanmugasundaram K, Nayak BK, Friedrichs WE, Kaushik D, Rodriguez R, Block K. NOX4 functions as a mitochondrial energetic sensor coupling cancer metabolic reprogramming to drug resistance. Nat Commun. 2017;8:997.

10. Barrett T, Wilhite SE, Ledoux P, Evangelista C, Kim IF, Tomashevsky M, et al. NCBI GEO: archive for functional genomics data sets-update. Nucleic Acids Res. 2013;41: D991-D995.

11. Kim D, Langmead B, Salzberg SL. HISAT: a fast spliced aligner with low memory requirements. Nat Methods. 2015;12:357-60.

12. Liao Y, Smyth GK, Shi W. featureCounts: an efficient general purpose program for assigning sequence reads to genomic features. Bioinformatics. 2014;30:923-30.

13. Maranchie JK, Zhan Y. Nox4 is critical for hypoxia-inducible factor 2- $\alpha$ transcriptional activity in von Hippel-Lindau-deficient renal cell carcinoma. Cancer Res. 2005;65:9190-3.

14. Asakuma J, Sumitomo M, Asano T, Asano T, Hayakawa M. Selective Akt inactivation and tumor necrosis factor-related apoptosis-inducing ligand sensitization of renal cancer cells by low concentrations of paclitaxel. Cancer Res. 2003;63:1365-70.

15. Shang D, Liu Y, Xu X, Han T, Tian Y. 5-Aza-2-deoxycytidine enhances susceptibility of renal cell carcinoma to paclitaxel by decreasing LEF1/phospho-beta-catenin expression. Cancer Lett. 2011;311:230-6.

16. Zhu H, Wang Z, Xu Q, Zhang Y, Zhai Y, Bai J, et al. Inhibition of STAT1 sensitizes renal cell carcinoma cells to radiotherapy and chemotherapy. Cancer Biol Ther. 2012;13:401-7.
17. Han T, Shang D, Xu X, Tian Y. Gene expression profiling of the synergy of 5-aza-2'-deoxycytidine and paclitaxel against renal cell carcinoma. World J Surg Oncol. 2012;10:183.

18. Jiang H, Ma N, Shang Y, Zhou W, Chen T, Guan D, et al. Triosephosphate isomerase 1 suppresses growth, migration and invasion of hepatocellular carcinoma cells. Biochem Biophys Res Commun. 2017;482:1048-53.

19. Kathagen-Buhmann A, Maire CL, Weller J, Schulte A, Matschke J, Holz M, et al. The secreted glycolytic enzyme GPI/AMF stimulates glioblastoma cell migration and invasion in an autocrine fashion but can have antiproliferative effects. Neuro Oncol. 2018. https://doi.org/10.1093/neuonc/noy117.

20. Li B, Qiu B, Lee DSM, Walton ZE, Ochocki JD, Mathew LK, et al. Fructose-1,6-bisphosphatase opposes renal carcinoma progression. Nature. 2014;513:251-5.

21. Vexler ZS, Wong A, Francisco C, Manabat C, Christen S, Tauber $\mathrm{M}$, et al. Fructose-1,6-bisphosphate preserves intracellular glutathione and protects cortical neurons against oxidative stress. Brain Res. 2003;960:90-98.

22. Bajic A, Zakrzewska J, Godjevac D, Andjus P, Jones DR, Spasic $\mathrm{M}$, et al. Relevance of the ability of fructose 1,6-bis(phosphate) to sequester ferrous but not ferric ions. Carbohydr Res. 2011;346: 416-20.

23. Diaz-Ruiz R, Averet N, Araiza D, Pinson B, Uribe-Carvajal S, Devin A, et al. Mitochondrial oxidative phosphorylation is regulated by fructose 1,6-bisphosphate. A possible role in Crabtree effect induction? J Biol Chem. 2008;283:26948-55.

24. Norma A, Ronald A, Teresa C. Fructose 1,6-bisphosphate: a summary of its cytoprotective mechanism. Curr Med Chem. 2016;23:4396-417.

25. Tao Q-f, Yuan S-x, Yang F, Yang S, Yang Y, Yuan J-h, et al. Aldolase B inhibits metastasis through Ten-Eleven Translocation 1 and serves as a prognostic biomarker in hepatocellular carcinoma. Mol Cancer. 2015;14:170.

26. Chen W, Wang Y, Zhao A, Xia L, Xie G, Su M, et al. Enhanced fructose utilization mediated by SLC2A5 is a unique metabolic feature of acute myeloid leukemia with therapeutic potential. Cancer Cell. 2016;30:779-91.

27. Liu H, Huang D, McArthur DL, Boros LG, Nissen N, Heaney AP. Fructose induces transketolase flux to promote pancreatic cancer growth. Cancer Res. 2010;70:6368-76. 